SCIREA Journal of Clinical Medicine

ISSN: 2706-8870

http://www.scirea.org/journal/CM

January 24, 2021

SCIREA

Volume 6, Issue 1, February 2021

\title{
A new type of isolation cap for the protection of nursing severe COVID-19 patient
}

\section{Yun Zhang ${ }^{1}$, Hongtao Liu ${ }^{2}$}

${ }^{1}$ Guangxi University of Traditional Chinese Medicine is attached to The Guangxi School of Traditional Chinese Medicine, China.

${ }^{2}$ The First Affiliated Hospital,Jinan University, 510632 Guangzhou, China.

Corresponding author: Hongtao Liu (1htgood@sina.com)

Adress: The First Affiliated Hospital,Jinan University, 510632 Guangzhou, China.

Acknowledgements:Thanks for Y Yidu City First People's Hospital of Hubei Province's support.

\begin{abstract}
Since the outbreak of novel coronavirus disease (COVID-19) in China, Chinese nurses have undertaken most of the tasks to nursing severe COVID-19 patients, so they take a huge risk of exposure. Therefore, personal prevention and protection are of great importance.This article will introduce advantages of a new type of isolation cap that is used to protect nurse through several methods, including design, analysis, and clinical comparative. The new isolation cap has advantages of good airtightness, anti-indentation, anti-fog and so on .We hope to introduce this isolation cap as soon as possible, so as to share our preventive experience with nurses around the world.
\end{abstract}


Acknowledgements:All subjects participating in the image acquisition signed the consent form.

Keywords: Isolation cap, protection, COVID-19, nurses

\section{Background}

Since December 2019, COVID-19 broke out in Wuhan, the capital of Hubei province in China. This epidemic disease spread to all parts of the country rapidly and swept some countries and regions in the world. For patients with severe respiratory failure, tracheal intubation and mechanical ventilation can effectively improve hypoxemia[1]. The coronavirus is mainly spread via droplets[2]. During nursing severe COVID-19 patients, nurses are more likely to be exposed to high concentrations of virus droplets that are carried by patients. According to statistics on the website of National Health and Health Commission, before February 1, 2020, 1,716 medical workers were diagnosed with this virus in China, with 6 people died[3]. The protection is extremely important. This study introduces a new type of isolation cap from its design, usage and comparison with traditional masks and goggles and summarizes its advantages for the protection of medical staffs.

\section{Materials}

The new type of isolation cap used in this study is produced by Henan TUOREN BEST Medical Device Co., Ltd. (http://www.tuoren.com) and the model is Type B. This product has been tested by Chinese testing institutions. The virus filtration efficiency is $98.6 \%$ and the bacterial filtration efficiency is $98.7 \%$ (Figure 1). The product is composed of shawl cap body, window, filter device and tightening band. The filter device consists of a protective cover, fixing belt and adjusting cap (Figure 2). The cap body is made of non-woven cloth. The window is made of ethylene-vinyl acetate copolymer. The protective cover and filter membrane are made of polyvinyl and polypropylene respectively. 


\section{XKS 讯科标准}

检 测 报 告

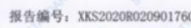

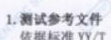

等

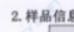

\begin{tabular}{|c|c|c|c|}
\hline H品名繁 & 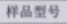 & 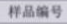 & 魴注 \\
\hline 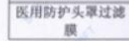 & 3 & $8=$ & 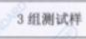 \\
\hline
\end{tabular}

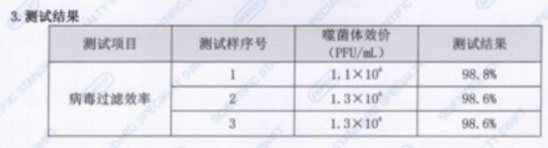

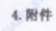

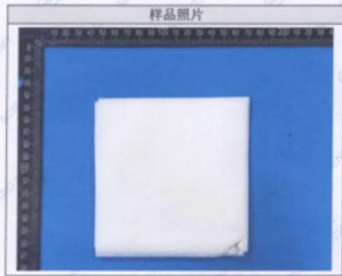

XK5) 讯科标准

检 测 报 告

报告据号: XKs2020R02240239C

1. 带抎费考文件

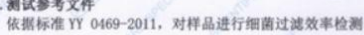

2. 样品信息

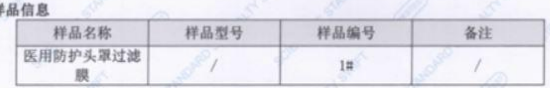

3. 溇识结果

\begin{tabular}{|c|c|c|c|}
\hline 勫识顷目 & 样品編号 & 标准要求 & 讨诘詓果 \\
\hline 细苚过： & $1=$ & $\geqslant 95 \preccurlyeq$ & $98.07 x$ \\
\hline
\end{tabular}

4. 的件

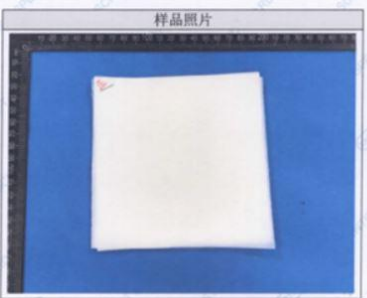

Figure 1.Test Report

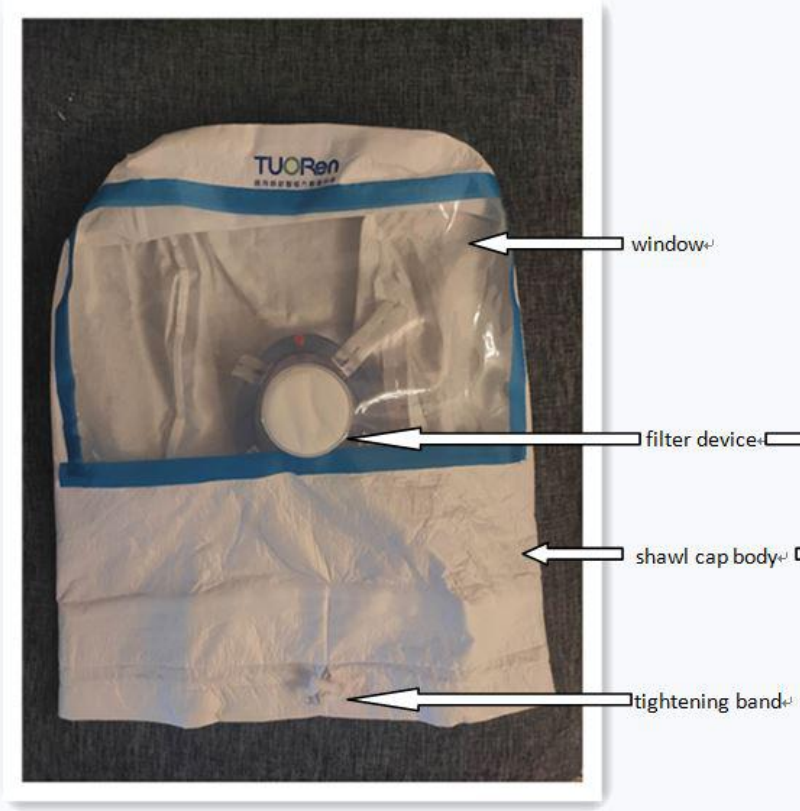

Outside

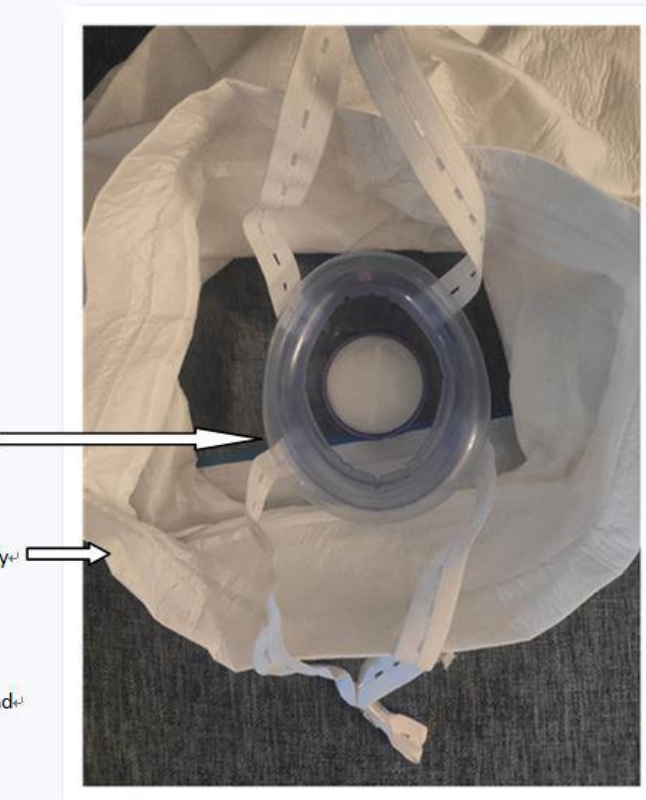

Inside

Figure 2.The new type of isolation cap 


\section{Usage}

Firstly, take out the isolation cap, put nose and mouth on the protective cover, pull the fixing behind your head, and adjust the cap to an appropriate position, so the protective cover can seal the nose and mouth. Then put the cap on the head backwards and tighten tightening band. Finally, you should wear protective clothing. (Figure 3).
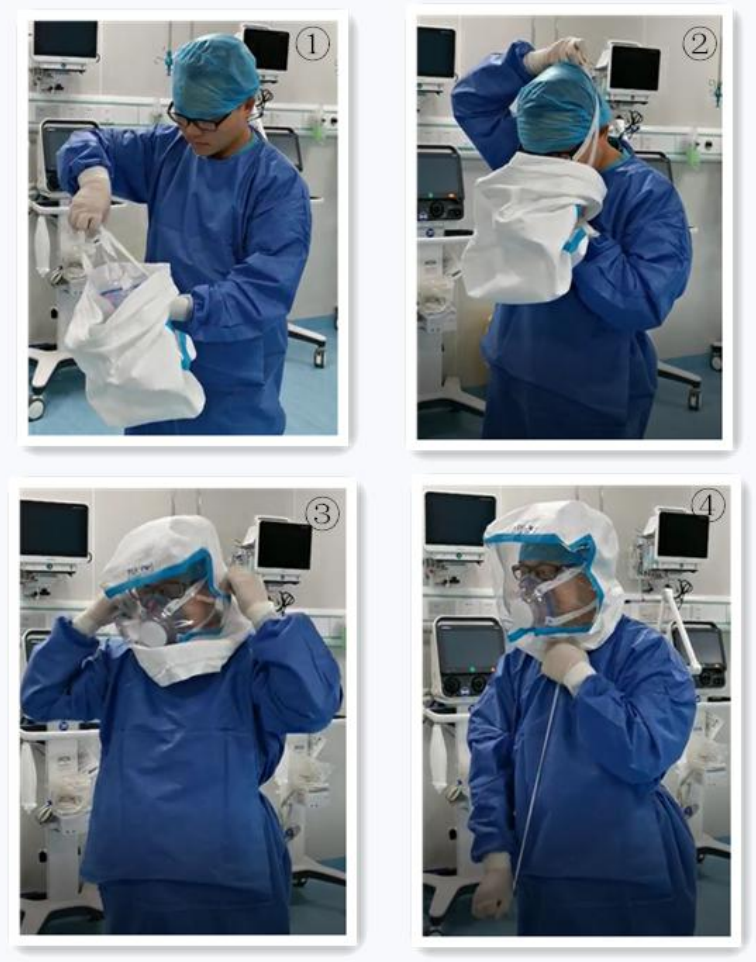

Figure 3.Usage of isolation cap

\section{Advantages}

\subsection{Good airtightness}

Traditional goggles are hard-link to the contours around the eyes, and they are not tightly sealed, which can easily lead to virus infection (Figure 4). Even with the anti-additive face screen, the head and face cannot be completely protected (Figure 5). The new isolation cap adopts an integrated design, which has better airtightness. After the adjusting cap is tightened, the head and face can be completely protected. 


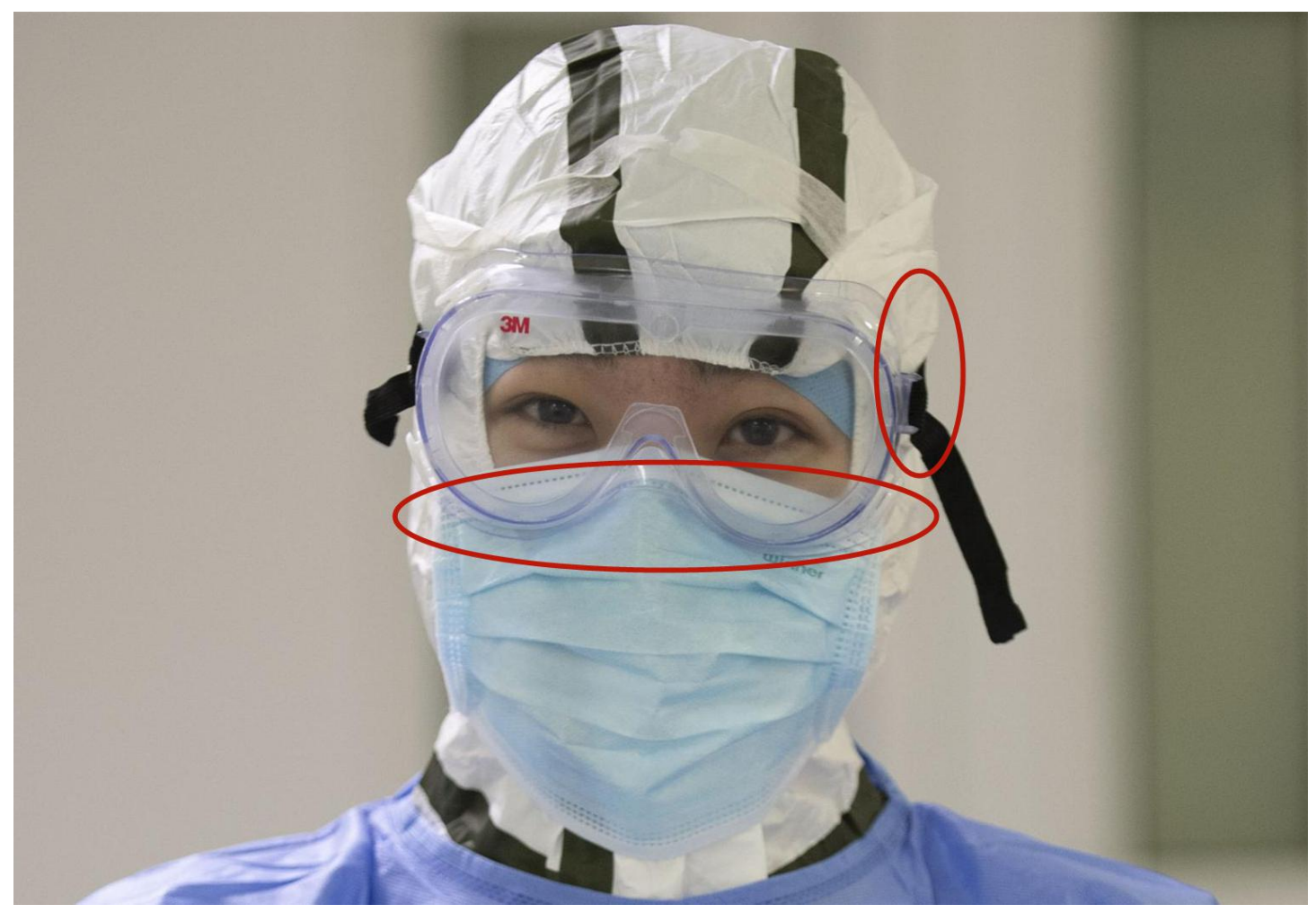

Figure 4.Disadvantages of goggles

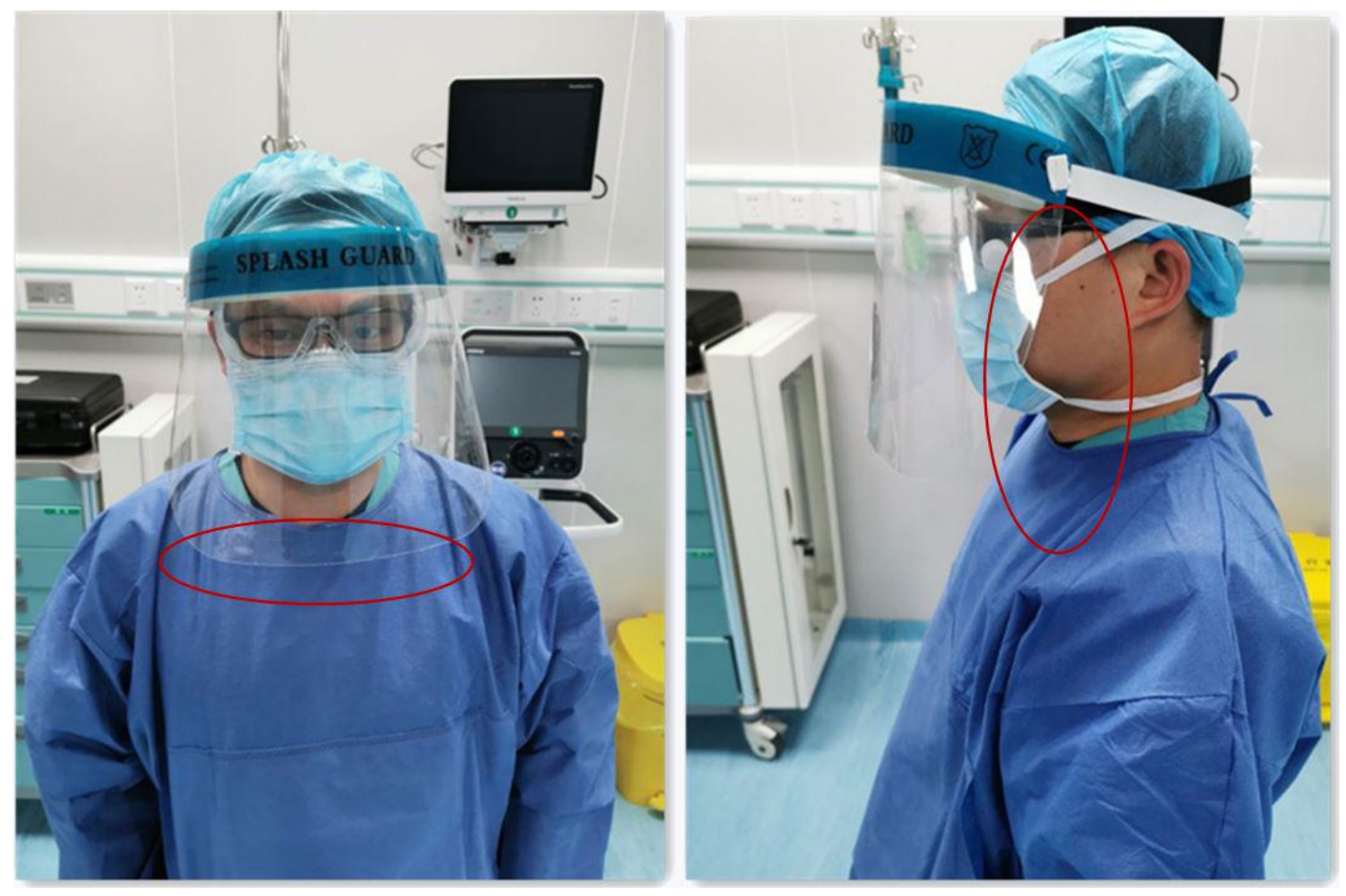

Figure 5.Disadvantages of face screen 


\subsection{Prevent indentation}

After the outbreak of COVID-19 in Wuhan, medical workers need do their jobs in isolation wards for a long time. Wearing goggles for a long time often causes indentations and even bruises the nose bridge and face (Figure 6). The new isolation cap has no goggles. Goggles is replaced by a wide-view perspective window, so there is no pressure around the eyes' areas, which can basically solve the problem of indentation around eyes. The airbag design is also used around the protective cover. It is soft and comfortable to wear and has good airtightness. In addition, it can reduce the pressure on the nose bridge and avoid the indentation. Therefore, this new isolation cap greatly deal with the problem of indentation.

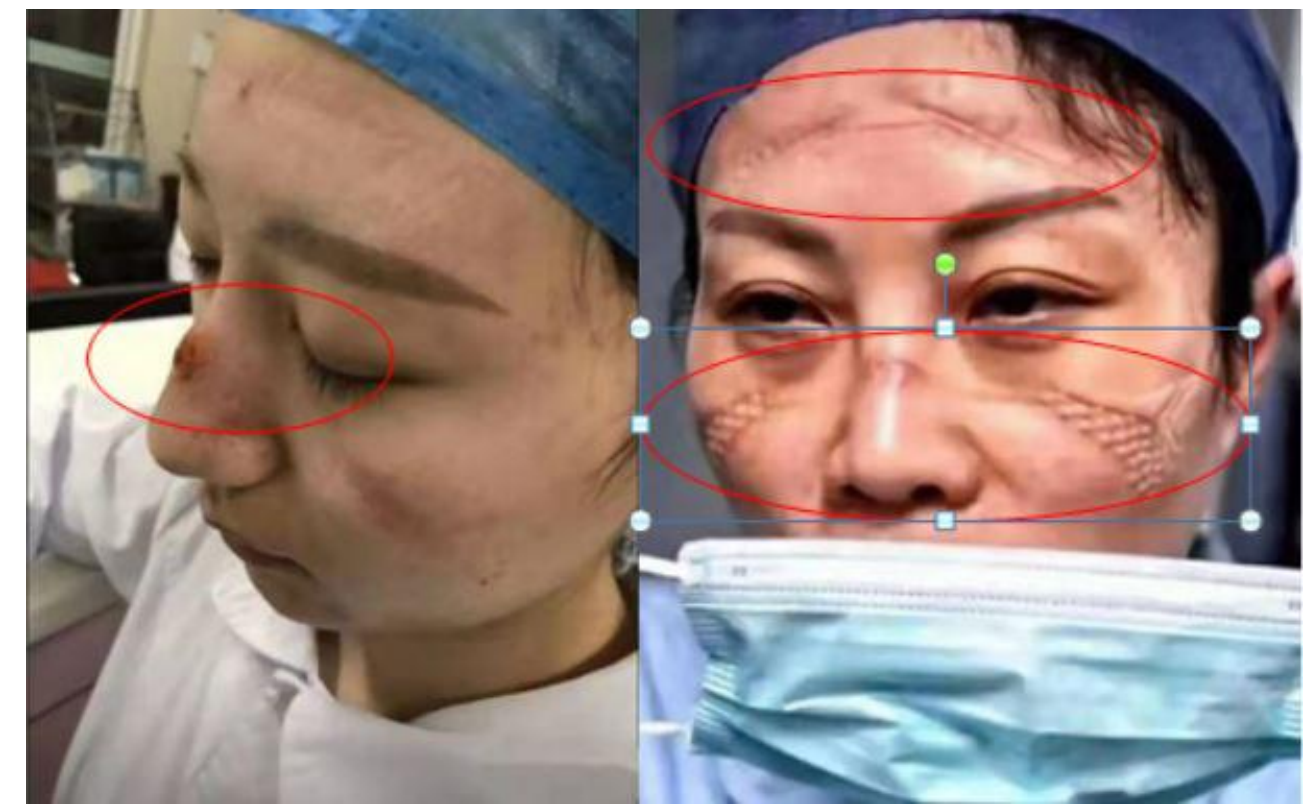

Figure 6. Indentation

\subsection{Antifogging}

After wearing protective gear, one of the biggest problem medical staff need to face is the fogging problem inside goggles, which seriously affects medical staff's vision and increases the difficulty of clinical operation. The goggles and its contours are hard and tight-sealing, which can easily generate fog the inside goggles. Normally, goggles will generate fog within 1 minute, and it is difficult to ensure a clear vision. The new type of isolation cap is used to cover the mouth and nose with air airbags that has better airtightness and the exhaled air will not remain in the cover, which avoids the generation of mist. In order to verify the anti-fog effect of the new isolation cap, we observed 10 medical staff From Yidu City First People's 
Hospital of Hubei Province(Approved by ethics committee of Yidu City First People's Hospital). They were asked to wear masks, goggles and this new isolation cap to intubate for severe COVID-19 patients in isolated wards (Adjust the room temperature to $23^{\circ} \mathrm{C}$ ). Then, observations were done to confirm whether it produce fog within half an hour or not. The fogging is defined as the visible mist. These results were analyzed by statistics with SPSS 21 . Chi-square and exact probabilistic method were adopted to compare the difference between traditional protective gear and this new isolation cap. $P \leq 0.05$ was considered statistically significant. Statistical results (Table 1) show the anti-fog effect between traditional protective gear and the new isolation cap is significantly different. The fogging rate of mask and goggles is $80 \%$, while the fogging rate of the new isolation cap is $10 \%$. The anti-fog effect of the isolation cap within 2 hours is obviously better than that of the combination of masks and goggles. Due to the strict definition to fogging conditions in this experiment, one case in the isolation cap group has slight fogging problem, but have no effect on vision, because of the large screen design. However, in the mask and goggles group, the fog is more serious, which affect the visual field.

\section{Discussion}

During the process of treatment to severe COVID-19 patients, nurses play an important role. Therefore, protection is very important. The new isolation cap has advantages of good airtightness, anti-indentation, anti-fog and so on. It is also suitable for other medical staff who work in isolation wards, especially after the outbreak of COVID-19 globally [4]. We hope to share our experience with anesthesiologists around the world. However, due to the serious pandemic situation and short time, we only got domestic certification, instead of EU certification. There are few clinical data, and some designs need to get better improvement. In this study, the producer provided us with 10 sets of new isolation caps, so the test sample of anti-fog effect comparison is small and it can give rise to some errors, which need to be verified with further clinical studies.

\section{Acknowledgments}

The authors would like to thank Dr.Yang fan(Yidu City First People's Hospital) provide us with data and photos. 


\section{References:}

[1] National health commission. Prevention and control of new coronavirus pneumonia (5th edition) [EB/OL]. From http://www.gov.cn/zhengce/zhengceku/2020-02/22/content_5482010.htm

[2] Huang C, Wang Y, Li X, Ren L, Zhao J, Hu Y, et al. Clinical features of patients infected with 2019 novel coronavirus in Wuhan, China. Lancet. 2020;395:497-506.

[3] China NHCotPsRo. Notice of the General Office of the National Health and Health Commission on issuing the guidelines for the use of common medical protective products in the prevention and control of pneumonia infected by new coronavirus (trial). From http://www.nhc.gov.cn/xcs/zhengcwj/202001/e71c5de925a64eafbe1 ce790debab5c6.shtml

[4] Latest rolling update: WHO characterizes COVID-19 as a pandemic. From https://www.who.int/emergencies/diseases/novel-coronavirus-2019 